Grant Waterer ${ }^{1,2}$

grant.waterer@uwa.edu.au

\title{
Review
}

\section{What is pneumonia?}

The diagnosis of pneumonia is both simple and complex. Recent research is challenging our concept of pneumonia and radiological gold standards that have underpinned research for decades. In particular, the accuracy of chest radiographs in diagnosing pneumonia is now highly questionable when compared with computed tomography scans. Depending on the question being asked, pneumonia can be defined in clinical, pathological, radiological, or microbiological contexts, or frequently a combination of all of these. However, while the field is changing, until we have new studies defining pneumonia in new ways, clinicians can be reassured that existing guidelines based on "old" standards remain as valid as they have always been.

\section{Introduction}

I suspect most clinicians would feel they "know" what pneumonia is when they are treating a patient. However, being asked to provide an exact definition of pneumonia is complicated and depends on whether you take a pathological, radiological, microbiological or clinical viewpoint. Recent radiological and clinical research has also questioned long standing concepts of pneumonia, especially community-acquired pneumonia (CAP), and challenged the radiological "gold standard" of a chest radiograph.

Complicating things further is that the majority of cases of CAP are probably treated as an outpatient; however, studies of CAP are overwhelmingly focused on patients admitted to hospital. As radiological, pathological and microbiological tests are rarely done in the outpatient setting, the question of "what is pneumonia" is even more challenging in a non-hospitalised patient.

@ERSpublications

Recent research has challenged our concept of pneumonia. New studies will define pneumonia in new ways, but clinicians can be reassured that existing guidelines based on "old" standards remain valid. https://bit.ly/3kJiV2N
In this review, I will discuss the various approaches to defining pneumonia from the point of view of a clinician faced with deciding whether or not to apply one of the various pneumonia guidelines as the appropriate standard of care in their patient.

\section{Pathology}

The gold standard for diagnosis of pneumonia should be pathology, but it is exceptionally rare to make the diagnosis this way and usually it is only in fatal cases where tissue samples are available to do so. While pneumonia is almost universally associated with infection, pathologically pneumonia is any inflammatory process involving the alveolar spaces. Non-infectious causes of pneumonia include lipoid pneumonia, lymphocytic interstitial pneumonia, cryptogenic organising pneumonia,

Cite as: Waterer G. What is pneumonia? Breathe 2021; 17: 210087. 
nonspecific interstitial pneumonia (and all its various sub-types [1]) and acute interstitial pneumonia [2], to name just a few. While all are much rarer than infectious causes of pneumonia, it is important that clinicians remember that there are important non-infectious causes as this may be highly relevant in the patient who does not improve on standard antibiotic therapy but has a radiological infiltrate consistent with pneumonia. While many of these non-infectious syndromes have suggestive clinical or radiological features, they will often be misdiagnosed as infections for some time before a diagnosis is made. In most cases a pathological specimen is needed to make the diagnosis. However, as all of these non-infectious causes are comparatively rare, for the rest of this review I will confine the discussion to infectious causes of pneumonia.

While infection can spread to the lung via the systemic route, overwhelmingly pneumonia is caused by pathogens entering through the respiratory route. As a result, the pathology in pneumonia is a combination of both airway and alveolar inflammation, extending into the interstitial space. Historically where pneumonia was limited to areas immediately adjacent to the bronchi this was called "bronchopneumonia" to distinguish it from widespread alveolar and interstitial involvement with sub-lobar, lobar or multi-lobar disease [3]. Although the concepts of bronchopneumonia and lobar pneumonia are probably still meaningful clinically as more extensive disease is generally associated with worse prognosis [4], almost all the clinical studies that make up our data on appropriate treatment of pneumonia have not considered these as separate entities.

Macroscopically in the pre-antibiotic era pneumonia was described as having four stages. The first stage, consolidation, is characterised by the early inflammatory response with neutrophils, lymphocytes and fibrin. The second stage, red hepatisation, occurs 2-3 days after consolidation where the lungs become hyperaemic, with a consistency similar to liver. Grey hepatisation follows after a further 2-3 days and is a relatively avascular stage with lungs appearing grey-brown to yellow due to the fibro-purulent exudates and disintegration of red blood cells. The final stage, resolution, may last for many weeks as the inflammatory changes resolve and the lung slowly re-aerates.

At a histological level the initial pulmonary infiltrate is reflective of the immune response to the pathogen, with the predominant cells dependent on whether it is primarily a viral, bacterial, fungal or protozoal infection [3]. As well as the alveolar infiltrate, there is associated oedema and inflammatory response in the surrounding interstitial space. Over time macrophages become predominant, particularly in the resolution phase.

Given the difficulty in obtaining large enough lung samples to study the histopathology of pneumonia, most of our information comes from more indirect sampling through cytology of bronchoalveolar lavage and sputum samples. Without a confirmatory microbiological diagnosis, cytological findings consistent with pulmonary infection can only be considered suggestive of pneumonia, particularly given the broad array of non-infectious causes of inflammatory pneumonia already discussed. So, while in theory pathology is the "gold standard" for the diagnosis of pneumonia, it is not a useful concept clinically other than to understand the utility of other modes of diagnosis.

\section{Physiology}

There are no pathognomonic physiological changes in pneumonia. The inflammatory response is the same as infections elsewhere with mild disease associated with fever and malaise through to fulminant septic shock in severe cases. While septic shock is typically associated with bacteraemia, with greater levels of bacteria recovered from blood in those who develop shock [5], viral pathogens including severe acute respiratory syndrome coronavirus 2 (SARS-CoV-2) may also produce an identical phenotype [6]. An important concept is that in the setting of severe sepsis the inflammatory response itself directly damages the lung, typically manifesting as acute respiratory distress syndrome and/or diffuse alveolar damage. However, with the exceptions of coronavirus disease 2019 (COVID19) and pneumonia due to pneumocystis, antiinflammatory strategies have not been shown to be effective in reducing adverse outcomes in pneumonia.

If there is sufficient volume of lung involved in the infective process, then hypoxia may result through mismatch of ventilation and perfusion of the affected area(s). However, hypoxia is not universal and frequently absent in milder disease. Reduced lung compliance and increased work of breathing may also contribute to hypoxia in more severe disease [7]

\section{Clinical diagnosis}

The clinical presentation and findings of pneumonia, i.e. symptoms of cough, fever, dyspnoea, and crackles on examination, are suggestive, but not pathognomonic. Recency of symptoms is also a key component of the diagnosis, as a duration of several weeks or more raises the possibility of more chronic infections, particularly in areas where they are endemic (e.g. tuberculosis, melioidosis). Clinicians should also be aware that pneumonia may present atypically in the elderly (i.e. with confusion and lack of fever) and in patients with significant immunocompromise.

The issue of whether pneumonia can be diagnosed without any imaging confirmation is 
frequently debated [8]. In reality it depends on the resources available and how sick the patient is. When radiology is not available, the presence of fever and cough along with appropriate focal signs on examination (crackles, bronchial breathing, dullness to percussion, etc.) is clearly consistent with a diagnosis of pneumonia and should be treated as such. When the clinical features raise the possibility of competing diagnoses (cardiac failure, pleural effusion, pulmonary emboli, etc.), or comorbid illnesses make interpreting the clinical signs more problematic (e.g. underlying COPD, pulmonary fibrosis or bronchiectasis), then imaging should be obtained to confirm or refute the diagnosis of pneumonia. Equally if a patient is physiologically compromised enough to require hospitalisation, then radiology is mandatory to ensure the diagnosis is correct and that other pathology (e.g. empyema, lung abscess) is not present.

Numerous studies have shown that medical coding of pneumonia poorly correlates with proven radiological pneumonia [9, 10], strongly suggesting that clinicians over diagnose pneumonia. The reasons for this consistent trend towards overdiagnosis is complex, but both financial incentives favouring a diagnosis of pneumonia and the existence of clear guidelines and pathways to ensure evidence-based treatment are likely to be factors. In the setting of ventilator-associated pneumonia (VAP), clinical features alone have a very poor sensitivity and specificity [11].

The clinical assessment of severity of pneumonia is critical to deciding on the site of treatment and type of treatment that is appropriate. It is strongly recommended that one of the validated scoring systems is used in conjunction with clinical judgement, either the pneumonia severity index or the CURB-65 (confusion, urea, respiratory rate, blood pressure, age over 65 years) [12]. The presence of confusion, a respiratory rate greater than 30 breaths per min or a systolic blood pressure less than $90 \mathrm{mmHg}$ should always trigger significant concern as these have consistently been associated with much higher risks of adverse outcome in multiple studies over the past three decades.

\section{Radiology}

In the absence of pathology, a chest radiograph showing changes consistent with acute infection has been the diagnostic standard for pneumonia. While gross changes such as lobar or multi-lobar disease are generally consistently identified and agree upon, for lesser degrees of change (e.g. patchy infiltrates possibly consistent with bronchopneumonia) studies have shown only modest agreement between specialists reading the films in blinded fashion [13-15]. While not specifically reported in the literature, it is likely that there is a high degree of correlation between observers for classic lobar pneumonia with most disagreement likely in non-lobar disease with segmental or smaller infiltrates.

With the widespread adoption of chest computed tomography (CT), which provides a more accurate view of lung parenchyma than radiography, a further challenge has been made to the accuracy of the latter in the diagnosis of pneumonia [16, 17]. Claessens et al. [17], in a study of 319 patients with suspected CAP, found one-third of patients with a negative chest radiograph had infiltrates on CT consistent with pneumonia, while $30 \%$ of patients suspected of pneumonia based on the chest radiograph were cleared by the $\mathrm{CT}$ scan. SELF et al. [16] reported that, in 3423 patients undergoing CT and chest radiography, using CT as the gold standard chest radiography only had a sensitivity of $43.5 \%$ and a specificity of $93 \%$ for the diagnosis of pneumonia. Both of these studies raise significant concerns regarding the accuracy of chest radiography in the diagnosis of CAP, although it should be noted that the CT scans were always later than the plain radiology, biasing the results in favour of the later test as infiltrates often evolve over time.

The finding that CT scans find more pneumonia than chest radiographs was not surprising, but the proportion of cases in which this occurred was considerable, raising the question of whether $\mathrm{CT}$-positive, chest radiograph-negative pneumonia has the same pathogen mix and prognosis as chest radiograph-positive disease. The findings of UPCHURCH et al. [18] that there appeared to be no difference in pathogens, severity or outcome in $\mathrm{CT}$-positive, chest radiograph-negative, and chest radiograph-positive CAP is reassuring that this is not a different entity.

With respect to possible false positive diagnoses of pneumonia by chest radiogrpahy, a key part of the assessment relies on being able to discern acute from chronic changes. In patients with chronic pulmonary infiltrates, for example those with pulmonary fibrosis or bronchiectasis, nonpneumonic exacerbations are frequently diagnosed as CAP due to failing to recognise the long-standing nature of the radiological changes. Infiltrates related to COPD are also commonly misinterpreted as pneumonia [14]. Outside of the communityacquired setting this becomes significantly more problematic, especially in patients on mechanical ventilation in the setting of other acquired pulmonary disorders (e.g. acute respiratory distress syndrome, diffuse alveolar damage). Due to the difficulty in distinguishing acute from subacute or chronic pulmonary changes, in the setting of VAP the chest radiograph has usually only been a considered part of the diagnostic criteria rather than sufficient for the diagnosis [19]. One recent meta-analysis suggested the pooled sensitivity for chest radiography in the diagnosis of VAP was $88 \%$, with a specificity of only $26 \%$ [11]. Due to the increased difficulties in obtaining CT scans in ventilated patients, this mode of imaging is yet to 
significantly impact; however, increasing portability of scanners may alter this in coming years.

Pulmonary ultrasound is an emerging imaging technology that has expanded in its availability of point-of-care tools with increasingly high quality, reducing cost and lower levels of technical skill required to perform examinations. Several studies have suggested that lung ultrasound may have closer alignment with the clinician diagnosis of pneumonia [20] or CT findings [21] than chest radiography. Pulmonary ultrasound has also been suggested as being useful in the bedside diagnosis of VAP [22]. However, pulmonary ultrasound is still some way from being an accepted standard of care or an inclusion criteria for clinical studies, particularly given the absence of outcome studies and acknowledged difficulties in imaging some areas of the lung and problems with patients with larger body habitus. With continued improvements, clinician familiarity and the perceived relative advantage over chest radiography in terms of portability, time and radiation, it is possible that ultrasound may become a key imaging modality in the future.

\section{Microbiology}

With the notable exception of VAP (discussed later), microbiological identification of a respiratory pathogen has never been a requirement for the diagnosis of pneumonia and nor has it even been sufficient in the absence of consistent clinical and radiological features. All the pathogens that cause pneumonia can cause acute bronchitis and most can cause acute upper respiratory tract infections, so by definition their presence does not constitute a diagnosis of pneumonia. Equally even the most rigorous of studies fail to identify the pathogen in up to $50 \%$ of patients with pneumonia [23-25], either due to the impact of prior antibiotic use, the less than perfect efficacy of current tests, the difficulty in getting respiratory tract samples from conscious patients or possibly still unidentified pathogens we currently do not have diagnostic tests for. With respect to the last possibility, it should be remembered that human metapneumovirus, now recognised as a significant cause of CAP, was first identified in 2001 [26], and of course new viruses are emerging all the time, as SARS-CoV-2 has made the whole world aware.

The notable exception to the requirement for microbiology is in the diagnosis of VAP. As already noted, patients with VAP frequently have other causes for pulmonary infiltrates reducing the reliability of radiology as a gold standard. Further complicating the diagnosis of VAP is that common nosocomial pathogens will frequently be cultured from tracheal aspirates even in patients who do not go on to develop clinical pneumonia. For this reason, the sensitivity and specificity of qualitative cultures of respiratory secretions in isolation from other features of pneumonia (i.e. clinical, radiological) in ventilated patients for the diagnosis of VAP is relatively poor $[19,27]$. However, since clinical indicators of pneumonia also have a poor predictive ability in this setting [11], a combination of clinical, radiological and microbiological features is required to be confident of the diagnosis [19]. There has been significant debate for decades over the role of quantitative or semi-quantitative culture methods and the cut-offs required for the diagnosis of pneumonia. While values of $<10^{3}$ colony forming units (CFU) per $\mathrm{mL}$ for specimens obtained by protective specimen brush or $<10^{4} \mathrm{CFU}$ per $\mathrm{mL}$ from samples obtained by bronchoalveolar lavage are generally not suggestive of pneumonia, no specific values have been identified that are sufficient to over-ride clinical decision making based on clinical features of the patient [19]. The same is also true of semi-quantitative cultures of endotracheal aspirates.

\section{Other diagnostic tests}

Laboratory findings consistent with pneumonia include a raised peripheral white cell count, raised platelet count and elevated $\mathrm{C}$-reactive protein (CRP), but none of these are particularly sensitive or specific. A variety of biomarkers have been studied in the setting of pneumonia, with the most well-known being procalcitonin. While an elevated procalcitonin is supportive of a diagnosis of pneumonia, it may be normal when the infecting pathogen is viral, an "atypical" pathogen such as Mycoplasma pneumoniae or Legionella and in the setting of a bacterial secondary infection following influenza [28, 29]. Systemic inflammatory response is also often reduced in VAP or hospital-acquired pneumonia (HAP), further reducing the utility of procalcitonin. For these reasons procalcitonin is not recommended as a diagnostic test in the setting of CAP [12], nor in VAP or HAP [19].

With respect to CRP, it has similar limitations to procalcitonin, but is also raised by a variety of non-infectious inflammatory processes [30], further limiting its utility as a diagnostic test in pneumonia. While a very high CRP may increase the probability of pneumonia being present, this is not the case in most patients [31]. Erythrocyte sedimentation rate (ESR) has similar limitations to CRP [30], and also has limited diagnostic utility in pneumonia. Both CRP and ESR are generally higher in bacterial pneumonia than viral pneumonia, but there is considerable overlap and neither can be relied on to rule out bacterial infection. In summary, all the laboratory tests discussed can support the diagnosis of pneumonia, but none are sufficient to include or exclude it or able to accurately identify the pathogen.

\section{Conclusion}

For an extremely common and serious disease, the diagnosis of pneumonia remains challenging, 
with abundant published data suggesting clinicians are not particularly accurate based on radiological gold standards. Furthermore, traditional chest radiography gold standards are now under assault by CT studies showing up to $40 \%$ of cases may be misdiagnosed when called or not-called pneumonia.

Clinicians should, however, be reassured that much of the speculation over "what is pneumonia" is at this point more of academic than practical importance. All of our pneumonia guidelines are based on studies that used inclusion and exclusion criteria that clinicians recognise. Until new studies are carried out that show that new ways of making the diagnosis lead to better outcomes (either better patient outcomes or possibly better antibiotic stewardship outcomes), all of the "old" studies remain valid. The main conclusion that clinicians should draw over all the uncertainty about how to most accurately diagnose pneumonia is just that, there is uncertainty. Therefore, if you have diagnosed pneumonia in a patient and they are not responding appropriately, the diagnosis should be reconsidered. Similarly, if there are atypical features, do not be tempted to diagnose pneumonia just because that makes it easier to decide on what treatment path to follow. The over-diagnosis mistake has created many problems we are only starting to tackle with good antibiotic stewardship programmes. As COVID-19 has focused the world's attention on pulmonary infection, we can hope that all the significant advances in imaging and microbiological studies lead to significant new insights into how to more accurately diagnose pneumonia over the coming decade [32].

\section{Affiliations}

\section{Grant Waterer ${ }^{1,2}$}

${ }^{1}$ School of Medicine and Pharmacology and Lung Institute of Western Australia, University of Western Australia, Perth, Australia. ${ }^{2}$ Northwestern University, Chicago, IL, USA.

\section{Conflict of interest}

G. Waterer has nothing to disclose.

\section{References}

1. Travis WD, Costabel U, Hansell DM, et al. An official American Thoracic Society/European Respiratory Society statement: update of the international multidisciplinary classification of the idiopathic interstitial pneumonias. Am J Respir Crit Care Med 2013; 188: 733-748.

2. Taniguchi $\mathrm{H}$, Kondoh Y. Acute and subacute idiopathic interstitial pneumonias. Respirology 2016; 21: 810-820.

3. Kumar V, Abbas A, Fausto N, et al. Robbins and Cotran Pathologic Basis of Disease. 7th Edn. Philadelphia, Elsevier Saunders, 2005

4. Waterer GW. The diagnosis of community-acquired pneumonia. Do we need to take a big step backward? Am Respir Crit Care Med 2015; 192: 912-913.

5. Rello J, Lisboa T, Lujan M, et al. Severity of pneumococcal pneumonia associated with genomic bacterial load. Chest 2009; 136: 832-840.

6. Gu X, Zhou F, Wang Y, et al. Respiratory viral sepsis: epidemiology, pathophysiology, diagnosis and treatment. Eur Respir Rev 2020; 29: 200038.

7. Light RB. Pulmonary pathophysiology of pneumococcal pneumonia. Semin Respir Infect 1999; 14: 218-226.

8. Wootton D, Feldman C. The diagnosis of pneumonia requires a chest radiograph ( $\mathrm{x}$-ray) - yes, no or sometimes? Pneumonia (Nathan) 2014; 5: Suppl. 1, 1-7.

9. Guevara RE, Butler JC, Marston BJ, et al. Accuracy of ICD9-CM codes in detecting community-acquired pneumococcal pneumonia for incidence and vaccine efficacy studies. Am J Epidemiol 1999; 149: 282-289.

10. Aronsky D, Haug PJ, Lagor C, et al. Accuracy of administrative data for identifying patients with pneumonia. Am J Med Qual 2005; 20: 319-328

11. Fernando SM, Tran A, Cheng W, et al. Diagnosis of ventilatorassociated pneumonia in critically ill adult patients - a systematic review and meta-analysis. Intensive Care Med 2020; 46: 1170-1179.
12. Metlay JP, Waterer GW, Long AC, et al. Diagnosis and treatment of adults with community-acquired pneumonia. An official clinical practice guideline of the American Thoracic Society and Infectious Diseases Society of America. Am J Respir Crit Care Med 2019; 200: e45-e67.

13. Moncada DC, Rueda ZV, Macias A, et al. Reading and interpretation of chest X-ray in adults with communityacquired pneumonia. Braz J Infect Dis 2011; 15: 540-546.

14. Hopstaken RM, Witbraad T, van Engelshoven JM, et al. Interobserver variation in the interpretation of chest radiographs for pneumonia in community-acquired lower respiratory tract infections. Clin Radiol 2004; 59: 743-752.

15. Albaum MN, Hill LC, Murphy M, et al. Interobserver reliability of the chest radiograph in community-acquired pneumonia. PORT Investigators. Chest 1996; 110: 343-350.

16. SelfWH, Courtney DM, McNaughton CD, et al. High discordance of chest $\mathrm{x}$-ray and computed tomography for detection of pulmonary opacities in ED patients: implications for diagnosing pneumonia. Am J Emerg Med 2013; 31: 401-405.

17. Claessens YE, Debray MP, Tubach F, et al. Early chest computed tomography scan to assist diagnosis and guide treatment decision for suspected community-acquired pneumonia. Am J Respir Crit Care Med 2015; 192: 974-982.

18. Upchurch CP, Grijalva CG, Wunderink RG, et al. Communityacquired pneumonia visualized on CT scans but not chest radiographs: pathogens, severity, and clinical outcomes. Chest 2018; 153: 601-610.

19. Kalil AC, Metersky ML, Klompas M, et al. Management of adults with hospital-acquired and ventilator-associated pneumonia: 2016 clinical practice guidelines by the Infectious Diseases Society of America and the American Thoracic Society. Clin Infect Dis 2016; 63: e61-e111.

20. Bourcier JE, PaquetJ, Seinger $M$, et al. Performance comparison of lung ultrasound and chest x-ray for the diagnosis of pneumonia in the ED. Am J Emerg Med 2014; 32: 115-118. 
What is pneumonia?

21. Cortellaro F, Colombo S, Coen D, et al. Lung ultrasound is an accurate diagnostic tool for the diagnosis of pneumonia in the emergency department. Emerg Med J 2012; 29: 19-23.

22. Mongodi S, Via G, Girard M, et al. Lung ultrasound for early diagnosis of ventilator-associated pneumonia. Chest 2016 149: 969-980.

23. Jain S, Self WH, Wunderink RG, et al. Community-acquired pneumonia requiring hospitalization among U.S. adults. N EnglJ Med 2015; 373: 415-427.

24. Jain S, Williams DJ, Arnold SR, et al. Community-acquired pneumonia requiring hospitalization among U.S. children. N EnglJ Med 2015; 372: 835-845.

25. Holter JC, Muller F, Bjorang O, et al. Etiology of communityacquired pneumonia and diagnostic yields of microbiological methods: a 3-year prospective study in Norway. BMC Infect Dis 2015; 15: 64 .

26. van den Hoogen BG, de Jong JC, Groen J, et al. A newly discovered human pneumovirus isolated from young children with respiratory tract disease. Nat Med 2001; 7: 719-724.
27. Cavalcanti AB, Zampieri FG, Rosa RG, et al. Hydroxychloroquine with or without azithromycin in mild-to-moderate Covid-19. N Engl J Med 2020; 383: 2041-2052.

28. Self WH, Balk RA, Grijalva CG, et al. Procalcitonin as a marke of etiology in adults hospitalized with community-acquired pneumonia. Clin Infect Dis 2017; 65: 183-190.

29. Musher DM, Bebko SP, Roig IL. Serum procalcitonin level, viral polymerase chain reaction analysis, and lower respiratory tract infection. J Infect Dis 2014; 209: 631-633.

30. Lapic I, Padoan A, Bozzato D, et al. Erythrocyte sedimentation rate and C-reactive protein in acute inflammation. Am J Clin Pathol 2020; 153: 14-29.

31. Minnaard MC, de Groot JAH, Hopstaken RM, et al. The added value of $\mathrm{C}$-reactive protein measurement in diagnosing pneumonia in primary care: a meta-analysis of individual patient data. CMAJ 2017; 189: E56-E63.

32. Waterer GW. Applying the lessons learned from coronavirus disease 2019 to improve pneumonia management. Curr Opin Infect Dis 2021; 34: 175-179. 